\title{
CARACTERIZAÇÃO TEMPORAL DA PRECIPITAÇÃO PLUVIAL DO MUNICÍPIO DE PORTO VELHO/RO NO PERÍODO DE 1945 A 2003
}

\author{
Temporary characterization of the rainfall in Porto Velho/RO in the period of 1945 to 2003
}

\author{
Ricardo Braz Bezerra \\ Graduado em Geografia pela FUFRO- UNIR \\ Mestre em Meteorologia pela UFCG \\ Professor da UNICENTRO - Jaru/RO \\ Porto Velho/RO - Brasil \\ rcabrazz@gmail.com
}

Renilson Targino Dantas

Graduado em Meteorologia pela UFPB

Doutor em Agronomia pela UEPJM/SP

Professor Adjunto da UFPB e UFCG

Campina Grande/PB - Brasil renilson@dca.ufpb.br

Avenildson Gomes Trindade

Graduado em Geografia pela FUFRO- UNIR

Mestre em Geociência e Meio Ambiente pela UNESP

Professor da UNICENTRO - Jaru/RO

Porto Velho/RO - Brasil

avenildson@gmail.com

RESUMO: A pesquisa analisou o comportamento das precipitações pluviais em Porto Velho, para o período de 1945 a 2003, mostrando a tendência da precipitação pluviométrica dentro da série temporal, e sua variabilidade interanual nos 59 anos e os números de frequência de ocorrência de todos os eventos dentro da faixa de variabilidade estabelecida graficamente. As principais características do regime pluviométrico do município de Porto Velho são a sua sazonalidade bem definida em um período chuvoso que se estende de novembro a abril com precipitação mensal, oscilando entre $228,9 \mathrm{~mm}$ a 329,6 mm e um período de estiagem de junho a setembro com precipitação mensal, oscilando entre $38,7 \mathrm{~mm}$ a 107,7 mm, sendo os meses de maio e outubro considerados meses de transição de um regime para outro. A tendência da precipitação pluvial de Porto Velho em número de ocorrência está dentro da normalidade, pois, a maioria dos eventos encontrados ficou dentro da faixa de variabilidade estabelecidas sendo somente dezesseis ocorrências fora da faixa de variabilidade. A análise da distribuição da variabilidade trimestral da série histórica dos eventos de precipitação pluvial também não houve uma tendência para acréscimo ou decréscimo dos valores médios trimestral, pois um percentual de magnitude significante de $62,7 \%$ a $79,78 \%$ ocorreu entre a faixa de variação da medida de dispersão. Os anos e trimestre de precipitações pluviais que tiveram anomalias coincidiram com os fenômenos El Niño e La Niña.

Palavras-chaves: Análise estatística. Sazonalidade. Tendência da precipitação. Faixa de variabilidade. 
ABSTRACT: Actually, we have studied some procedures of the local rain in Porto Velho, Rondonia, With regard to the period 1945 - 2003, including the survey of the rainfall according to the shower and the series of occurrences on all events, in general. The main characteristics of the rainfall condition here are sue has. A rainy weather from Number to April with monthly rainfall between 228,9 mm, and 329,6 mm, and winter time from June to September with monthly rainfall between 38,9 mm, and 107,7 mm, and may and October are taken as transition months in the period. The tendency of pluvial precipitation in Porto Velho has been regular according to the number of occurrences as only 16 of them are out of parameters. The analysis of the distribution of the quarterly variability of the historical series of rain water, there was no quarterly tendency on the increase or decrease of medium values as there is a percentage of meaning magnitude between $62,7 \%$ and $79,78 \%$ in relation to dispersion measure. The period of years and quarters of rain water out of the variability of the dispersion measure has kept up with the phenomena El Niño and La Niña.

Keywords: Statistical analysis. Seasonality. Tendency of the rainfall. Variability strip.

\section{INTRODUÇÃO}

O estudo climatológico das diversas variáveis do tempo atmosférico é de extrema importância, tendo em vista o impacto ambiental que a anomalia desses componentes provoca no clima regional. Análises dos comportamentos das séries climáticas de alguns, ou até mesmo de todos esses elementos, visando destacar possíveis periodicidades existentes, são fundamentais para o planejamento de inúmeras atividades hidrológicas, em especial as de drenagem de águas pluviais, agrícolas, econômicas, sociais, de nível de reservatórios de barragem de hidroelétricas e dentre outras.

A variável precipitação é importante na caracterização do clima e assim, interfere em todos os segmentos das atividades econômicas e na relação do ecossistema através do ciclo hidrológico, pois a água encontra-se em todos os estágios ou fases no sistema superfície-atmosfera, que tem sua variabilidade temporal e espacial relacionamento com elementos e fatores climático local, regional e global e, consequentemente torna relevante seu estudo como mostra os trabalhos de AGUIAR (1995), BEZERRA (2004) e MARENGO (2001).

As condições climáticas do Estado de Rondônia, particularmente do Município de Porto Velho, são caracterizadas principalmente por chuvas abundantes. Tais condições se intensificam nos últimos e primeiros meses do ano, onde o fator de como ocorrem às preci- pitações anuais de valores extremos são fundamentais para o dimensionamento de qualquer tipo de projeto que considerem a precipitação pluviométrica com umas das variáveis de dimensionamento do projeto. Este artigo apresenta um diagnóstico do comportamento das variabilidades da precipitação pluviométrica interanual observadas às frequências ocorridas dentro de um intervalo de classe estabelecido em uma série histórica de 59 anos no período de 1945 a 2003.

Dentre as técnicas estatísticas para análise e interpretação de dados climáticos encontra-se a geoestatística, que tem como característica principal analisar a distribuição temporal da precipitação pluviométrica anual por meio de distribuição de frequência comum e relativa simples e acumulada, medidas de tendência e medidas de dispersão.

A carência de estudos da variabilidade espaçotemporal da precipitação pluviométrica do município de Porto Velho ocorre devido a falta de uma rede de estação climatológica de superfície, que se estenda pelos $34.082,0 \mathrm{~km}^{2}$ de área do município (IBGE 2003), sendo o mesmo coberto por apenas uma estação oficial de superfície registrada no Instituto Nacional de Meteorologia - INMET. Outro fato é a curta série de registros de dados dos elementos climáticos tendo seu início em 1945, até os dias atuais, sendo que o ideal para trabalhos mais consistentes na verificação de tendências cíclicas seriam séries espaço temporal de mais de 100 anos. 


\section{METODOLOGIA E PROCEDIMENTOS}

Para o desenvolvimento desta pesquisa foi utilizado dado de precipitação anual do período de 1945 a 2003 da estação climatológica de superfície de número 82825 , a $95,00 \mathrm{~m}$ em relação ao nível do mar com coordenadas de $08,26^{\circ} \mathrm{S}$ e $63,05^{\circ} \mathrm{W}$, fornecido pelo Instituto Nacional de Meteorologia - INMET, para a análise estatística da série de dados de precipitação pluviométrica.

\section{Materiais}

Dos totais de 59 anos dos dados de precipitação, foi feito o tratamento estatístico das medidas de dispersão (Variância, desvio padrão e coeficiente de variação), medidas de tendências e distribuição de frequências, na verificação das tendências climáticas compararam-se com a normal climatológica do período 45/03 com as normais climatológicas por décadas $(50,60,70,80$ e 90$)$ na verificação do comportamento de variação da precipitação dentro da série histórica.

\section{Métodos}

Os dados foram agrupados utilizando-se a distribuição de frequência e obedecendo à sequência cronológica mensal (soma dos valores diários) e anual (somatório dos totais mensais). Em seguida, determinaram-se as medidas de tendência central: média aritmética, mediana, de dispersão, amplitude e desvio padrão (s). As médias aritméticas mensais e anuais ( $\bar{x})$ e, os respectivos desvios padrões, foram calcula-

dos utilizando-se as expressões abaixo e a mediana, para dados agrupados, usando-se as frequências acumuladas.

$$
\begin{gathered}
\bar{x}=\sum_{i=1}^{N}\left(\frac{x_{i}}{N}\right) \\
s=\sqrt{\sum_{i=1}^{N} \frac{\left(x_{i}-x^{-}\right)}{(N-1)}}
\end{gathered}
$$

sendo: $x i=$ total mensal ou anual de chuva $(\mathrm{mm}) \mathrm{e}$ $\mathrm{i}=$ número de observações $(1,2, \ldots \mathrm{N})$.
Para efetivação das análises, ordenaram-se os dados anuais de chuvas em ordem crescente, sendo as probabilidades empíricas determinadas mediante a equação:

$$
\operatorname{Pr}(\%)=\frac{N}{n+1}
$$

Sendo: $\operatorname{Pr}=$ a probabilidade de ocorrência da chuva, em \%;

$\mathrm{N}=$ número de ordem dos dados agrupados; $\mathrm{n}=$ número total de anos da série.

\section{Medidas de dispersão}

Mediadas de dispersão representam à dispersão ou a variabilidade dos dados em torno do valor central, a média mais a amplitude que é a diferença entre os valores extremos. Em nosso estudo iremos utilizar as medidas de dispersão para a utilização da variabilidade anual da precipitação anual o desvio padrão, a variância e coeficiente de variância.

$$
\begin{gathered}
C V=\frac{S}{\bar{X}} \\
\mathrm{~S}^{2} \rightarrow \text { variância amostral }
\end{gathered}
$$

Os desvios de precipitação foram obtidos da subtração da média do cinquenta e nove anos de precipitação pela precipitação anual da série temporal que foi de 1945 a 2003 ano a ano, gerando desvios positivos e desvios negativos. Dado pala equação;

$\mathbf{D P}=\mathbf{P A}-\mathbf{P M}$, onde: $\mathbf{D P}=$ desvio de precipitação; $\mathbf{P A}=$ precipitação anual e $\mathbf{P M}=$ precipitação média. Em planilha no Excel.

A faixa de variabilidade da precipitação pluvial foi determinada pelo cálculo do desvio padrão dos 59 anos de dados pluviométricos, mostrando o comportamento da pluviometria anual dentro da série de 1945 a 2003, sendo que os desvios mais significativos considerados foram os que se encontram acima ou abaixo da faixa de variabilidade estabelecida.

As chuvas trimestrais foram calculadas com a utilização da planilha Excel, onde se somou a pre- 
cipitação mensal nas sequências: JFM, FMA, MAM, AMJ, MJJ, JJA, JAS, ASO, SON e OND.

A pluviometria por década dentro da série de 1945 a 2003 foi estabelecida pela média aritmética simples iniciando pela década de $45 / 54$ e terminado com a década de 95/03, tendo como base de análise a normal climatológica da série histórica em estudo e a variabilidade entre as décadas.

\section{Caracterização da Área de Estudo}

O município de Porto Velho, capital do Estado de Rondônia situa-se no extremo norte do estado e a sua área urbana é de aproximadamente de $150 \mathrm{~km}^{2}$, possui uma população estimada de 382.829 habitantes e densidade demográfica de $11,23 \mathrm{hab} . / \mathrm{km}^{2}$, para uma área de 34.082 $\mathrm{km}^{2}$ (IBGE, 2010). Segundo a classificação climática de Köppen o clima é tropical chuvoso do tipo Awi com uma estação relativamente seca nos meses de junho a agosto, denominada regionalmente como "verão", seu regime pluviométrico anual é de $2.286,6$, sendo os três primeiros meses do ano como os mais chuvosos e os meses de junho, julho e agosto como os menos chuvosos, contribuído para um período de grande flamabilidade é grande ocorrência de queimadas.

A temperatura média mensal vária de $25,2^{\circ} \mathrm{C}$ a $26,6^{\circ} \mathrm{C}$, sendo o mês de setembro o mais quente. A umidade relativa média mensal do ar, nunca é inferior a $80 \%$, porém nos meses de junho a agosto a umidade relativa do ar diária chega a 20\%. A cobertura vegetal é do tipo floresta ombrofila densa e aberta, com predominância de palmeiras, trepadeiras lenhosas, epífitas, árvores de médio e grande porte, floresta de cipó, bambu e sorocaba. O tipo de solo predominante no município são Latossolos Distróficos, Glei Distrófico, Podzólico Vermelho Amarelo Distrófico e os solos Litólicos Distróficos (RONDÔNIA, 2001, EMBRAPA, 1999).O relevo do município é pouco acidentado, não apresentando grandes elevações ou depressões, com variações de altitudes que vão de 70 metros a pouco mais de 500 metros. A região situa-se no vale do rio Madeira, entre a planície amazônica e o planalto central brasileiro.

\section{RESULTADOS E DISCUSSÃO}

\section{Precipitação Mensal}

As principais características do regime pluviométrico do município de Porto Velho são a sua sazonalidade bem definida em um período chuvoso que se estende de novembro a abril com precipitação mensal oscilando entre $228,9 \mathrm{~mm}$ a $329,6 \mathrm{~mm}$, e um período de estiagem de junho a setembro com precipitação mensal oscilando entre $38,7 \mathrm{~mm}$ a 107,7 $\mathrm{mm}$, sendo os meses de maio e outubro considerados meses de transição de um regime para outro. As maiores precipitações médias mensais acima de 300 mm concentram-se nos meses de dezembro a março

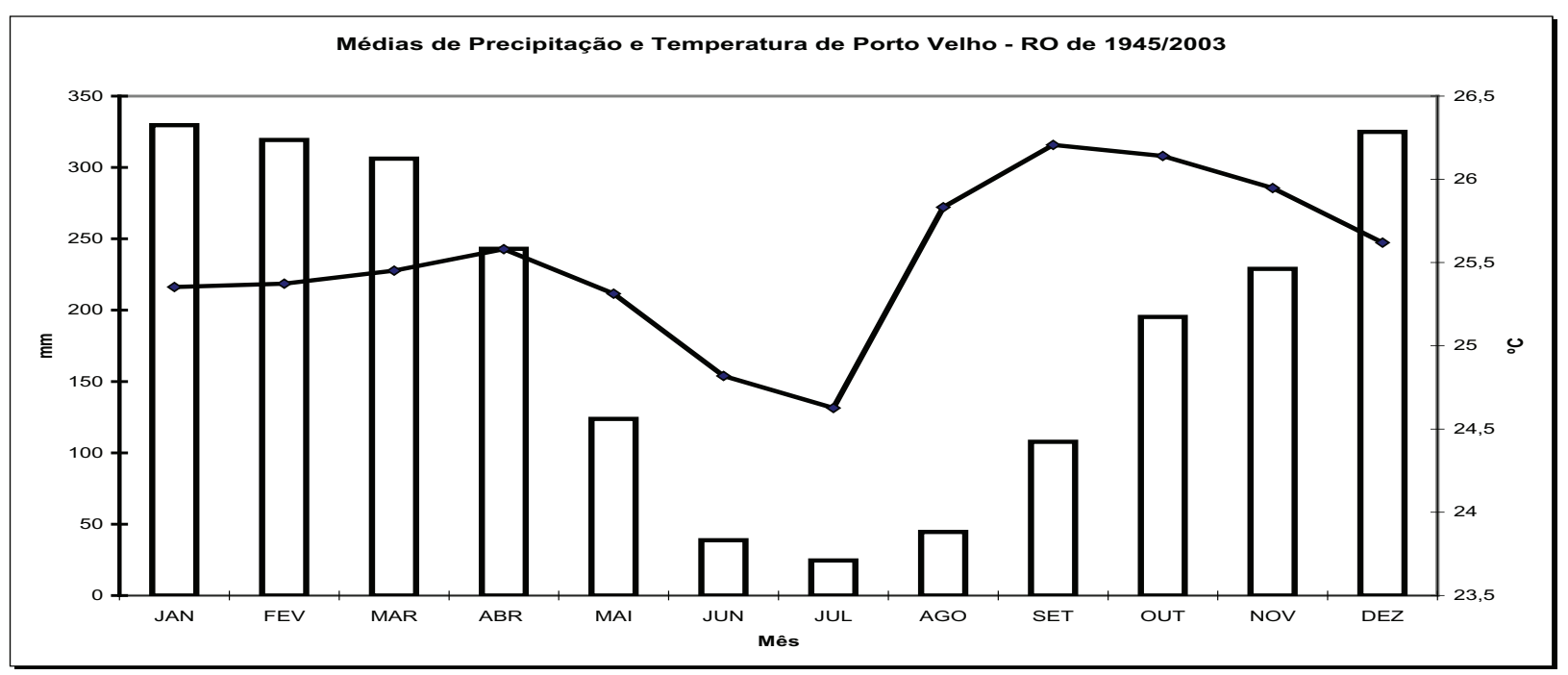

Figura 1: Média mensal da precipitação e temperatura média do ar de Porto Velho - RO de 1945/2003 
dentro da estação pluviométrica do município. A Figura 1 mostra o regime pluviométrico médio mensal e temperatura média mensal do ar a de 1945 a 2003.

Conforme estudo de NÓBREGA (2008) que analisou a distribuição da precipitação média da bacia hidrográfica do Rio Jamari, obtendo uma variação de 1.400 a 2.600 mm/ano. Já dados de precipitação média anual do período de 1999 a 2006, das cidades de Ariquemes, Cacoal, Porto Velho e Ji-Paraná do boletim climático da SEDAM (2006) registraram os respectivos resultados: $2.056,7 \mathrm{~mm} / \mathrm{ano} ; 1.543,7 \mathrm{~mm} /$ ano; $2.089,4 \mathrm{~mm} /$ ano e $1.898,7 \mathrm{~mm} /$ ano, os mesmo tem sazonalidade regida por sistema atmosférico de meso-escala, pois o período chuvoso concentra-se de dezembro a março e o de estiagem nos meses de junho a agosto, com precipitação mensal inferior a $50 \mathrm{~mm}$.

O regime da temperatura e de precipitação anual do município é regido pelo movimento aparente do sol e pela posição da Zona de Convergência Tropical (ZCIT), pois conforme menciona QUADRO et al. (2004) os núcleos de precipitação migram da parte central do país, no verão austral para o setor noroeste da América do Sul no inverno austral, acompanhando a migração anual da convecção profunda. Segundo RAO E HADA(1990), localidades na região norte que se encontram no Hemisfério Norte, exibem o máximo de chuvas durante o inverno austral (junho, julho e agosto) e mínimo durante o verão austral (dezembro, janeiro e fevereiro).

Com relação à temperatura, (NIMER; BRANDÃO, 1989; NIMER, 1991) afirma que durante o inverno do Hemisfério Sul (HS) toda a zona Meridional da Região Norte, em especial o sudeste, é frequentemente invadida por anticiclones originários de altas latitudes, que atravessa a Cordilheira dos Andes ao sul do Chile. Alguns são excepcionalmente intensos, podendo chegar a provocar o fenômeno da friagem.

Segundo BEZERRA(1996) nos meses de junho a agosto, quando as temperaturas mínimas ficam entre $18,8^{\circ} \mathrm{C}$ a $20,1^{\circ} \mathrm{C}$, são as menores temperaturas mínimas em razão da invasão da massa de ar fria que influência nesses meses mencionados, que contribui para registramos as menores temperaturas médias nos meses de junho e julho com $24,8^{\circ} \mathrm{C}$ e $24,6^{\circ} \mathrm{C}$, conforme Figura 1.

\section{Variabilidade da Precipitação Trimestral}

A distribuição da precipitação em trimestral é um bom indicador do comportamento de concentração do regime pluviométrico anual de uma localidade de acordo com NOBRE E MELO (2001) e VAREJÃOSILVA (2001), pois a mesma mostra os trimestres de maior e menor concentração de chuva. A Figura 2 indica que o regime de precipitação média anual

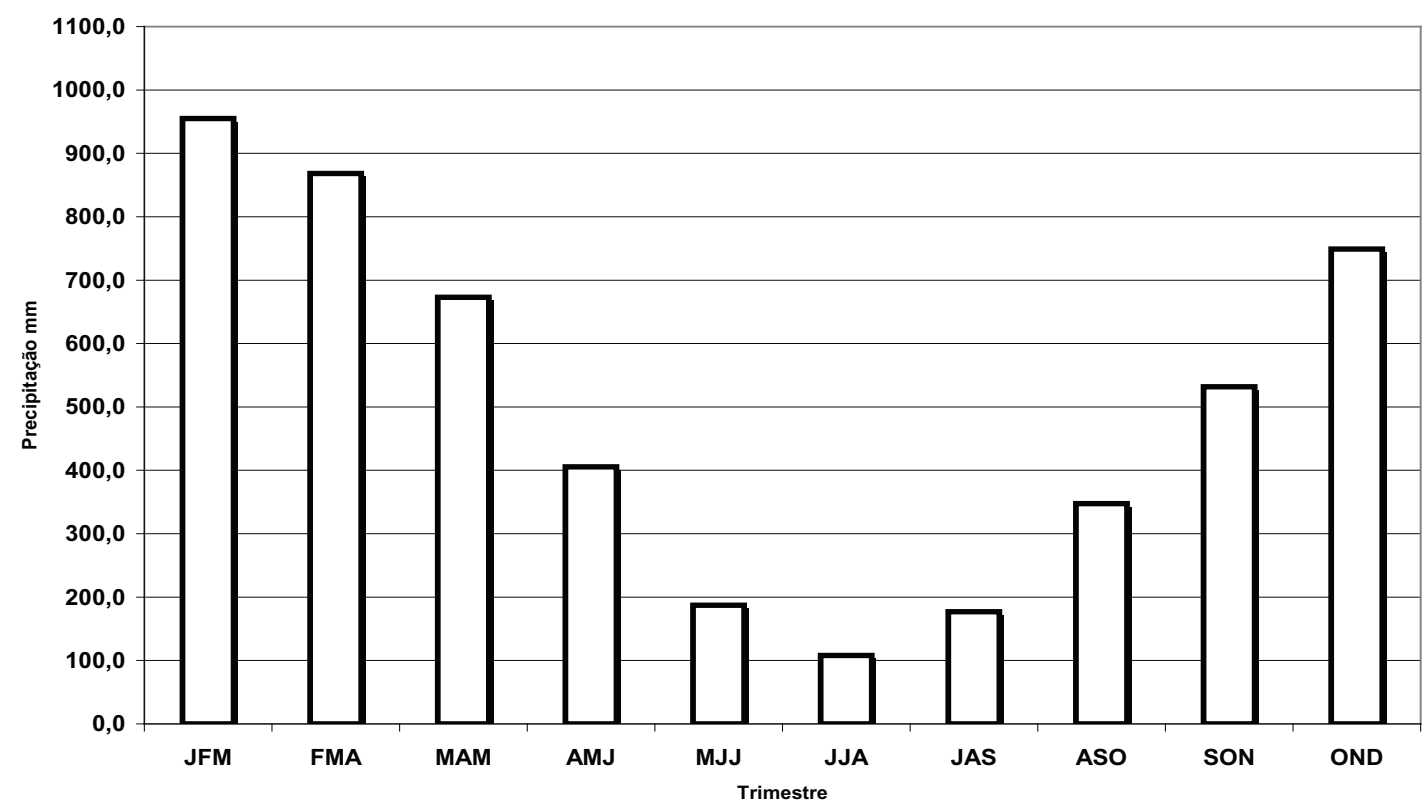

Figura 2: Precipitação trimestral de Porto Velho de 1945 a 2003 
entre os trimestres mais e menos chuvoso comportase como uma parábola com concavidade voltada para cima demonstrando que a distribuição da precipitação trimestral do município está bem definida em um período chuvoso e outro de estiagem, sendo que o trimestre com maior concentração de precipitação e o primeiro JFM com 1123,5 mm que corresponde 49,1\% da precipitação média anual da série histórica analisada de 1945 a 2003 que foi $2277,3 \mathrm{~mm}$ e o trimestre com menor concentração de precipitação é o sexto JJA com 107,9 mm, correspondendo 4,7\% da precipitação média da série histórica analisada.

Dos 59 anos analisados os extremos entre os trimestres mais chuvosos e menos chuvosos ocorrido acima da média histórica foram em 1968 com 285,4 $\mathrm{mm}, 1971 \mathrm{com} 481,1 \mathrm{~mm}$ e $1983 \mathrm{com}$ 1.410,6 mm em JFM e 1988 com 2,0 mm em JJA. Nesses anos as precipitações totais registradas foram de $2447,0 \mathrm{~mm}$ em 1968, 1912,6mm em 1971, 2381,3mm em 1983, e $1676,6 \mathrm{~mm}$ em 1988. Tendo como anomalia o mês de janeiro de 1971 que registrou somente $90,8 \mathrm{~mm}$, sendo que a média da série histórica para esse mês foi de $329,6 \mathrm{~mm}$.

O comportamento de cada trimestre dentro da série histórica segue a variabilidade intra-sazonal, que é regido por vários parâmetros meteorológicos que contribui para o aumento ou diminuição da precipitação trimestral, que não serão mencionados nesta pesquisa, pois a mesma está focando o comportamento da variabilidade da precipitação dentro da série histórica para a verificação da sua tendência ao longo dos cinquenta e nove anos pesquisados.

As Figuras 3 a 12 mostram o comportamento da variabilidade da precipitação do município de Porto Velho representada em dez trimestres que apresenta a tendência temporal dos desvios positivos (DP) e negativos (DN), dentro da faixa de variabilidade para mais ou para menos em relação à média da série. $O$ primeiro trimestre - JFM, conforme Figura 3 teve nove desvios positivos fora da faixa de variabilidade estabelecida, ocorridos nos anos de (1947, 1953, 1975, 1980, 1981, 1983, 1984, 1989 e 1994), com destaque para o ano de $1983 \mathrm{com}$ 455,5 mm de precipitação, os restantes dos anos citados os desvios de precipitação oscilaram entre $199,8 \mathrm{~mm}$ a $244,1 \mathrm{~mm}$ e oito desvios negativo fora da faixa de variabilidade estabelecida nos anos de (1964, 1968, 1971, 1978, 1985, 1987, 1995, 2002), tendo como destaque os anos de 1971 com 474,0 mm e 1987 com 407,2 mm

Na Figura 3 há um intervalo de 21 anos sem ocorrencia de desvio de precipitação positivo de 1953 a 1975 e entre eles um acentuado desvio negativo de precipitação ocorrido em 1971, que registro precipitação anual de 1912,6 mm. Conforme BEZERRA (2004, p. 55) ano de La Niña com desvio negativo anual de $374 \mathrm{~mm}$, abaixo da média anual de $2277,3 \mathrm{~mm}$. E com precipitação de 73,9 mm em janeiro, 196,5 mm em fevereiro e 210,7 em março de 1971, sendo que a "normal climatológica" dos meses em questão foram $329,6 \mathrm{~mm}, 319 \mathrm{~mm}$ e $306,1 \mathrm{~mm}$.

Percentualmente a distribuição temporal entre os desvios positivos e negativos da precipitação trimestral teve o seguinte comportamento: FMA com $16,9 \%$ de DP, $15,3 \%$ de DN e $67 \%$ na faixa da variabilidade (FV), MAM com $10,2 \%$ de DP, $13,6 \%$ de DN e $76,3 \%$ na FV, AMJ com 10,1\% de DP, $11,9 \%$ de DN e 78,0\% na FV, MJJ com $10,2 \%$ de DP, $10,2 \%$ de DN e 79,8\% na FV, JJA com $20,3 \%$ de DP, $15,3 \%$ de DN e 64,4 na FV, JAS com $18,6 \%$ de DP, $18,6 \%$ de DN e $62,8 \%$ na FV, ASO com $16,9 \%$ de DP, $18,6 \%$ de DN e $64,5 \%$ na FV, SON com $15,3 \%$ de DP, $18,6 \%$ de DN e $66,1 \%$ na FV e OND com $20,3 \%$ de DP, $16,9 \%$ de $\mathrm{DN}$ e $62,7 \%$ na FV.

A variabilidade da precipitação trimestral entre os desvios positivos e negativos, acima e abaixo da medida de dispersão ao longo dos 59 anos oscila entre $10,2 \%$ a $20,3 \%$ e $10,2 \%$ a $18,6 \%$ e a concentração da precipitação trimestral dentro da faixa de variabilidade intra-sazonal oscila entre $62,7 \%$ a $79,78 \%$ para os dez casos. Mostrando que as maiorias das variabilidades trimestrais encontram-se dentro da faixa da variação normal em relação da medida de dispersão utilizada no caso o desvio padrão.

As variações acima da média (VACm) e abaixo da média (VABm) dentro de cada trimestre comportaram-se da seguinte maneira: JFM com $49,2 \%$ VACm e $50,8 \%$ VABm, FMA com $52,5 \%$ VACm e $47,5 \%$ VABm, MAM com $49,2 \%$ VACm e $50,8 \%$ VABm, AMJ com $42,4 \%$ VACm e $57,6 \%$ VABm, MJJ com 37,3\% VACm e 62,7\% VABm, JJA com $37,3 \%$ VACm e $62,7 \%$ VABm, JAS com $45,8 \%$ 
3

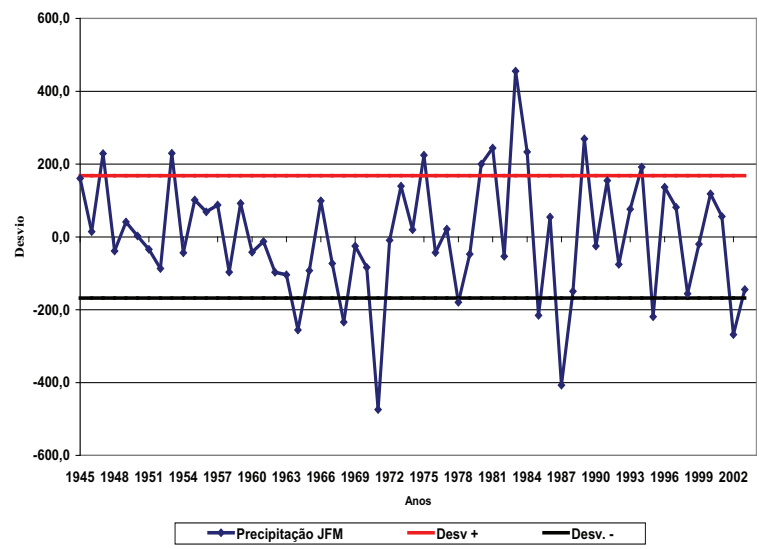

5

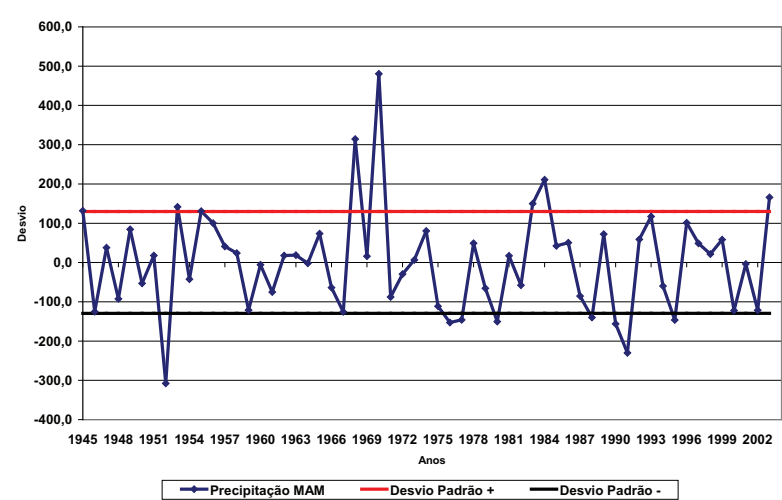

7

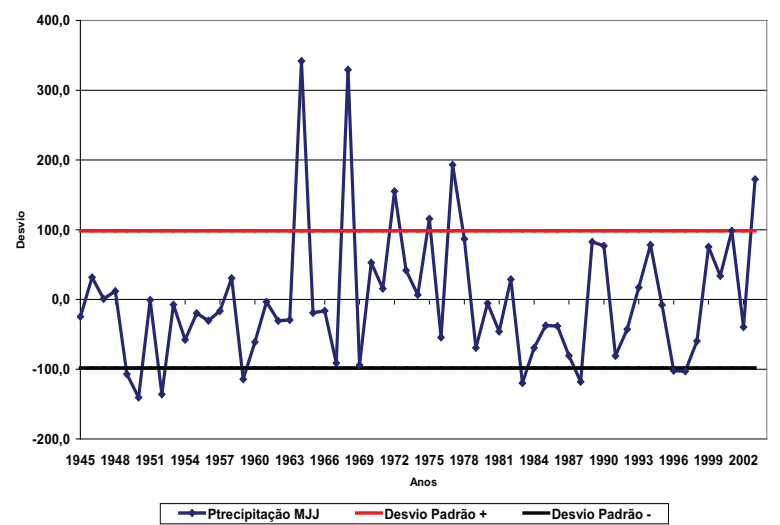

4

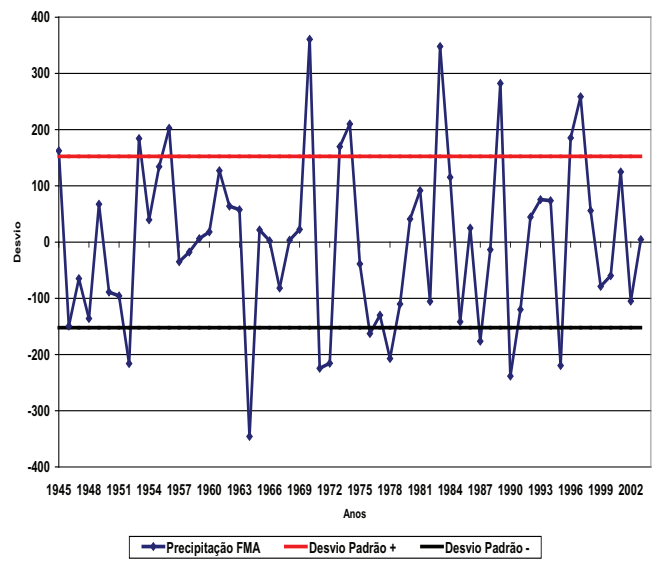

6

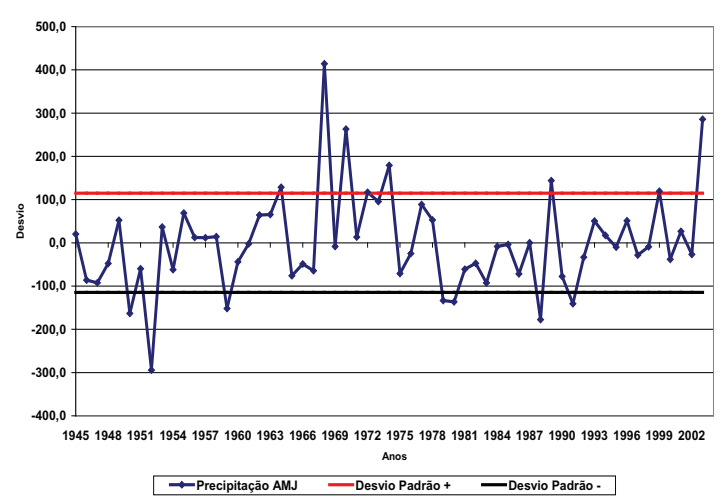

8

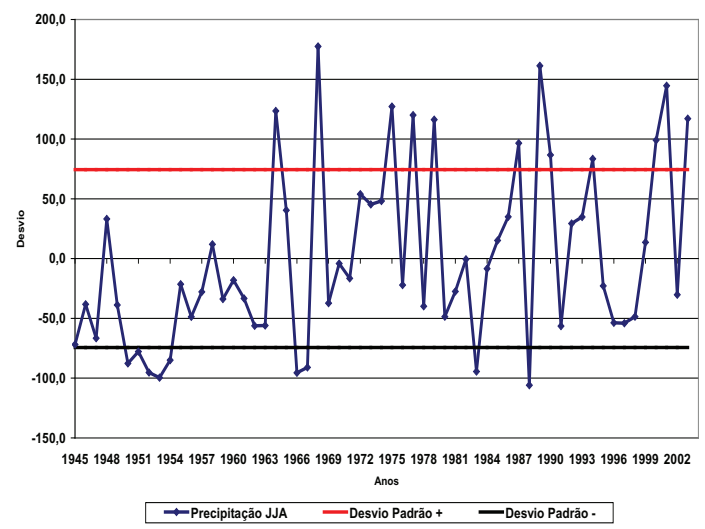


9

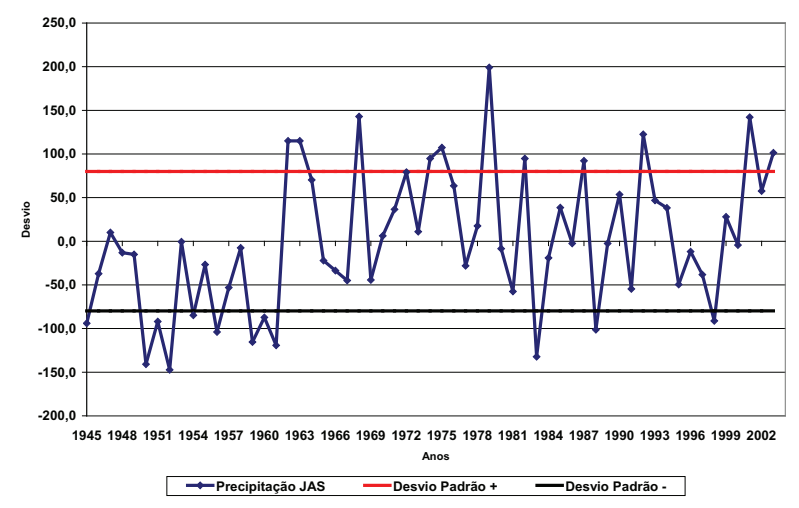

11

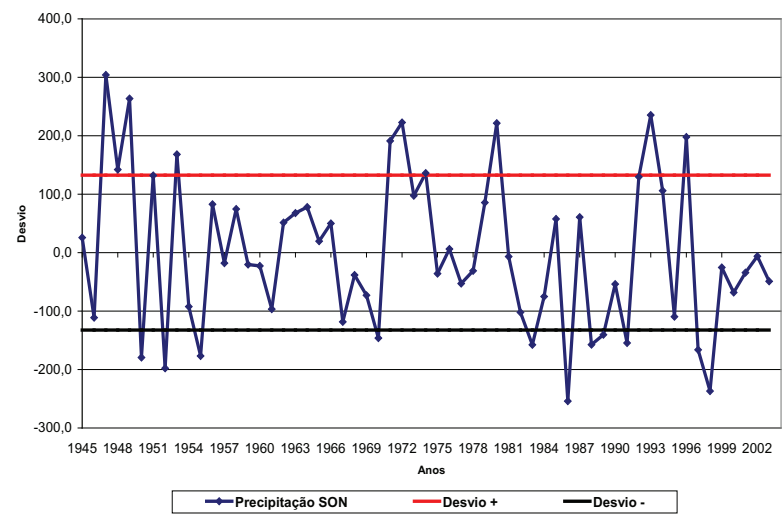

10

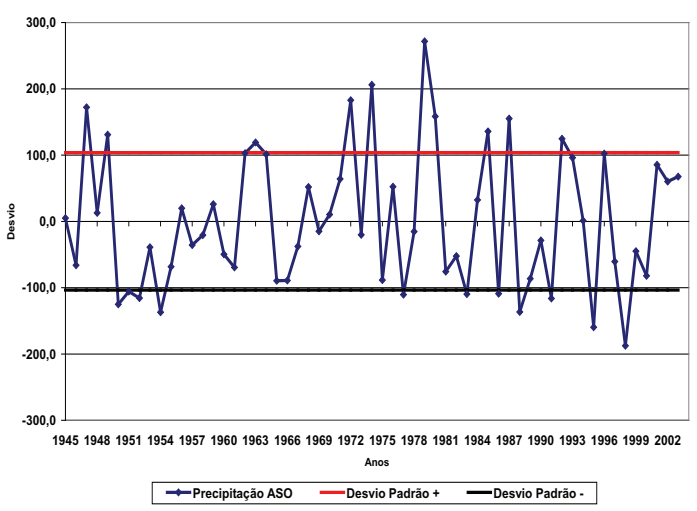

12

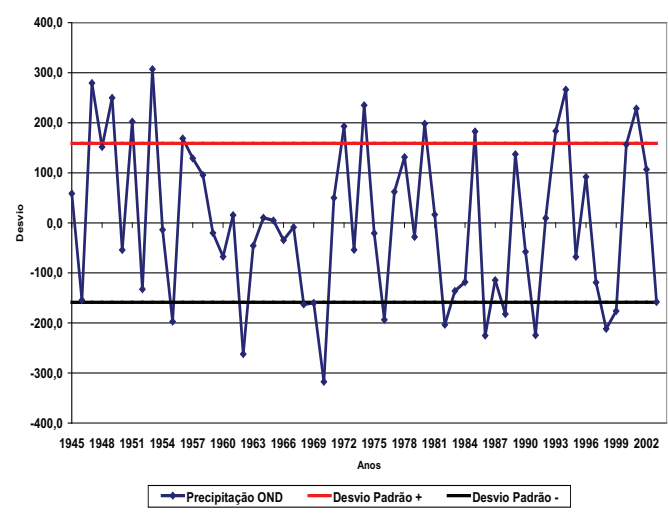

Figuras 3 a 12: Distribuições trimestrais da precipitação de Porto Velho de 1945 a 2003

VACM e 54,2\% VABm, ASO com 42,4\% VACm e $57,6 \%$ VABm, SON com $45,8 \%$ VACm e $54,2 \%$ VABm e OND com $47,5 \%$ VACm e $52,5 \%$ VABm. As amplitudes em milímetros entre os eventos extremos dos trimestres foram: 929,5 mm, 706,6 mm, 710,5 $\mathrm{mm}, 708,3 \mathrm{~mm}, 481,5 \mathrm{~mm}, 229,4 \mathrm{~mm}, 346,4 \mathrm{~mm}$, $459,3 \mathrm{~mm}, 558,2 \mathrm{~mm}$ e $621,7 \mathrm{~mm}$, seguindo a ordem de apresentação acima.

As Figuras 3 a 12 definem o comportamento da distribuição da variabilidade trimestral da série histórica analisada entre os eventos de precipitação, dos quais apresentam o seu comportamento peculiar, sendo que não há uma tendência para aumento ou diminuição dos valores médios de precipitação trimestral, pois um percentual significativo entre $62,7 \%$ a $79,78 \%$ ocorreu dentro da faixa de variação da medida de dispersão escolhida para os 59 anos da série histórica de 1945 a 2003.

\section{Variabilidade da Precipitação Interanual}

A Figura 13 mostra a variabilidade com desvios positivos e negativos em relação ao desvio padrão para mais e para menos como variável interanual dos 59 anos da série histórica e mostra que houve nove casos de precipitação acima da variável estabelecida e, sete casos abaixo da variável estabelecida, tanto para mais e para menos do desvio padrão que foi de 283,1 . Os anos de desvio positivos foram: (1947, com $426 \mathrm{~mm}$, 1949, com 328 mm, 1953, com 573 mm, 1972, com $380 \mathrm{~mm}, 1974$, com $528 \mathrm{~mm}, 1989$, com $548 \mathrm{~mm}$, 1993, com 357 mm, 1994, com 514mm, 2001, com $453 \mathrm{~mm}$ ) e os anos com desvio abaixo da variável estabelecida foram: (1950, com $356 \mathrm{~mm}, 1952$, com 661, 1971, com 374 mm, 1987, com 429 mm, 1988, com 610 mm, 1995, com, 346 mm, 1998, com 468 mm).

Na Figura 13 temos biênios de 1952/1953 e 


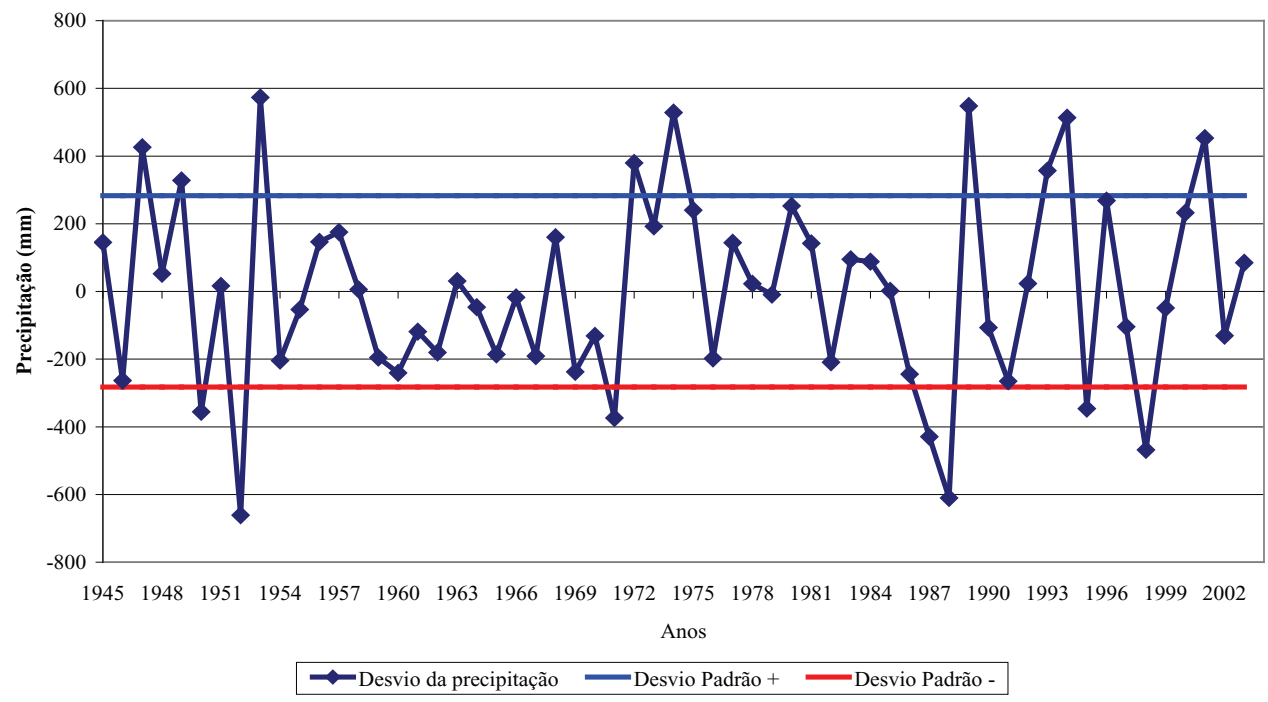

Figura 13: Variabilidade interanual de 1945 a 2003

1988/1989, os mesmos destacam-se, por apresentarem um desvio negativo em um ano e no ano seguinte registram desvio positivo em relação a normal climatológica da série, e dois períodos de comportamento de variabilidade interanual dentro da faixa da normalidade. O primeiro computou 17 anos estendendo-se de 1954 a 1970, com variabilidade inter-anual de amplitude curta e o segundo de 12 anos estendendo-se de 1975 a 1986, computando amplitude de variabilidade inter-anual mais longa, não dá para saber se o processo e cíclico, pois a série temporal que dispomos no momento não pode responder, pois precisaríamos de no mínimo de cem anos de dados. Porém, a Figura 13 mostra que a partir de 1987, as variabilidades inter-anuais com desvios para mais e para menos em relação a faixa de variabilidade estabelecida tiveram os maiores números de casos.

\section{Precipitação pluviométrica por década}

A chuva é o principal elemento da maioria dos projetos hidrológicos. Os problemas de engenharia relacionados com a hidrologia são em sua grande maior parte conseqüência de chuvas com grande intensidade ou volume e da ausência de chuva em longos períodos de estiagem. Chuvas de grande intensidade em áreas urbanas causam o alagamento das ruas, porque o sistema de drenagem não é projetado para chuvas muito intensas.
Segundo VILLELA e MATTOS (1975) precipitações de grande intensidade podem, ainda, causar danos à agricultura e a estrutura de barragens. A ausência de chuvas por longos períodos reduz a vazão dos rios, causando a diminuição do nível dos reservatórios. Vazões reduzidas devido à falta de chuva trazem danos ao ambiente do curso d'água, além de reduzir a água disponível para diluição de poluentes. A diminuição do nível dos lagos e reservatórios reduz a disponibilidade da água para usos como: abastecimento, irrigação e geração de energia. A umidade excessiva resultante de eventos de baixa intensidade e longa duração pode causar problemas à agricultura, reduzindo as colheitas.

É evidente, então que os problemas surgem quando a precipitação ocorre em situações extremas de intensidade, frequência, duração ou quando os intervalos entre precipitações são excessivamente longos.

Para MENDONÇA E DANNI-OLIVEIRA (2007) as características principais da precipitação são o seu total, duração e distribuições temporal e espacial. O total precipitado não tem significado se não estiver ligado a uma duração. Por exemplo, $100 \mathrm{~mm}$ pode ser pouco em um mês, mas é muito em um dia ou, ainda mais, em uma hora. A ocorrência da precipitação é processo aleatório que não permite uma previsão determinística com grande antecedência. O tratamento dos dados de precipitação para grande maioria dos problemas hidrológicos é estatístico conforme trabalhos de BACK (2001) e SILVA E RIBEIRO (2004). 


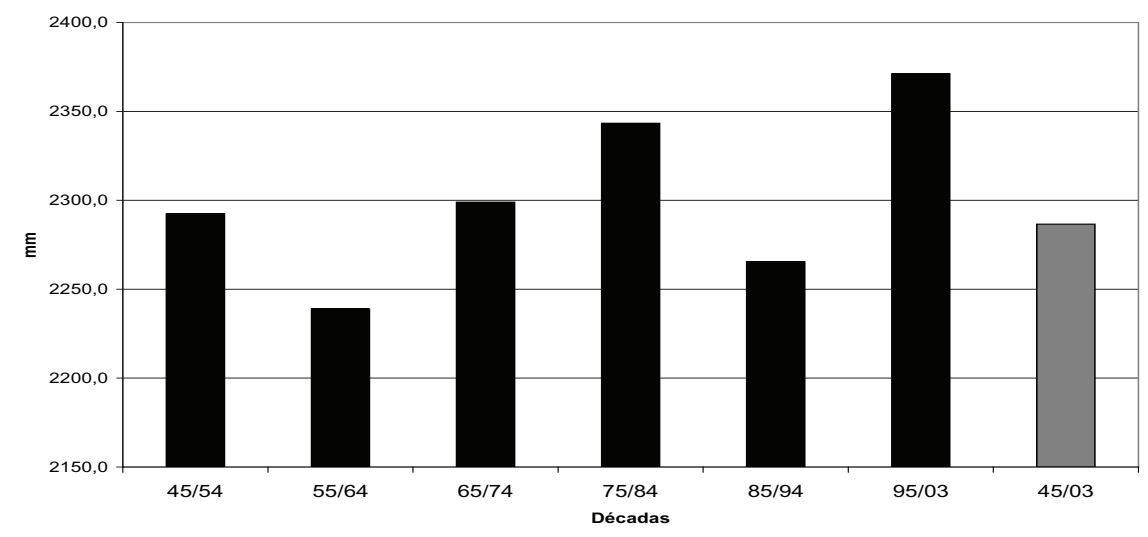

Figura 14: Distribuição da precipitação por décadas em Porto Velho de 1945/2003.tt

A década de 55/64 foi a mais seca em relação à média da série analisada, com valor de $47,7 \mathrm{~mm}$ amenos, e a mais chuvosa foi 95/03, com valor de 84,6 mm a mais, a variação da precipitação por década em relação à média de 45/03, oscila entre $12,4 \mathrm{~mm}$ a 84,6 $\mathrm{mm}$. mostrando que a variabilidade da precipitação por década no município de Porto Velho é quase inexpressiva, pois em termos percentuais oscilam entre $2,1 \%$ a 3,7\%. A menor precipitação ocorreu na década de 45/55, no ano de 1952, com precipitação de 1625,6 mm e a maior ocorreu na década de 85/94, no ano de 1989, com precipitação de $2834,6 \mathrm{~mm}$.

\section{Precipitação mensal por década}

A distribuição da precipitação mensal por década mostra o comportamento de uma série temporal de cada década em relação a normal climatológica dessa série analisada que foi de 1945 a 2003. Na Figura 15 verifica-se no mês de janeiro três situações onde a média mensal da precipitação foram maiores que a média mensal da normal climatológica escolhida e três casos onde a média da precipitação mensal por década foram menores que a normal climatológica, sendo que a oscilação da precipitação por décadas foi entre $(285,5 \mathrm{~mm}$ a

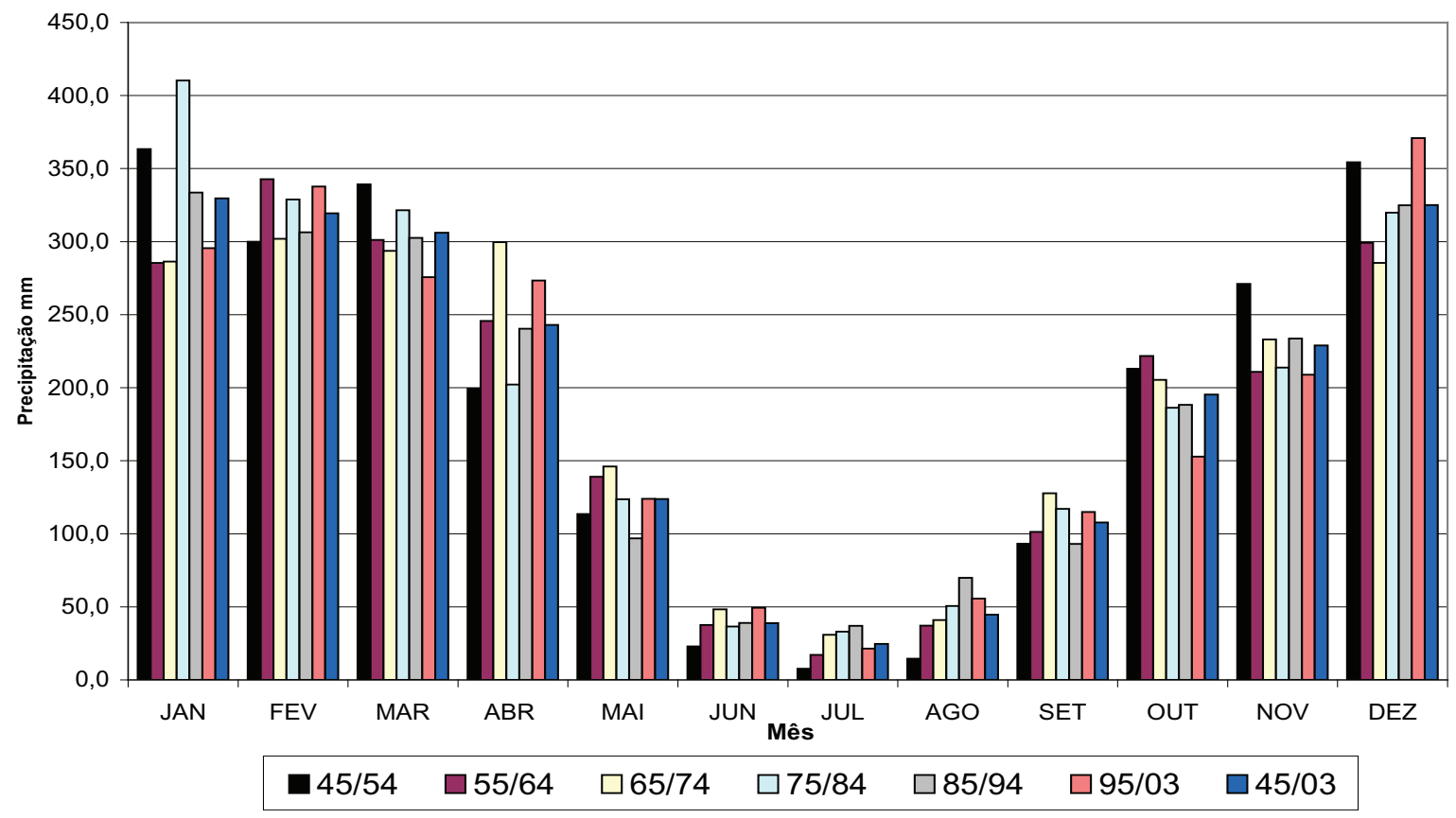

Figura 15: Distribuição da precipitação média mensal por décadas de 1945 a 2003 em relação à série histórica. 
$410,3 \mathrm{~mm}$, e a normal climatológica registraram $329,6 \mathrm{~mm}$ ). A diferencia na quantidade de precipitação para mais ou para menos de precipitação mensal por décadas no mês de janeiro em relação a normal climatológica foi de $80,7 \mathrm{~mm}$ e $58,4 \mathrm{~mm}$.

Outros meses de novembro e dezembro com destaque para os decênios 45/54 e 95/03, com variabilidade da precipitação mensal em relação a normal climatológica da série que não ultrapassam $46 \mathrm{~mm}$, mostrando que a diferença para mais o para menos em relação à média da série histórica escolhida não são discrepante

\section{Precipitação Anual}

A Tabela 1 está representada os valores totais de chuva anual e se verifica que dos 59 anos da série histórica só houve sete ocorrência de precipitação abaixo de $2000 \mathrm{~mm}$ e os restantes dos anos da série a precipitação foi superior a $2000 \mathrm{~mm}$.

Tabela 1: Valores totais de chuva anual de Porto Velho - RO (1945 - 2003).

\begin{tabular}{l|l|l|l|l|l|l|l|l|l|l}
\hline Anos & $\mathbf{0}$ & $\mathbf{1}$ & $\mathbf{2}$ & $\mathbf{3}$ & $\mathbf{4}$ & $\mathbf{5}$ & $\mathbf{6}$ & $\mathbf{7}$ & $\mathbf{8}$ & $\mathbf{9}$ \\
\hline 1940 & & & & & & 2.431 & 2.024 & 2.713 & 2.339 & 2.615 \\
\hline 1950 & $* 1.931$ & 2.303 & $* 1.626$ & 2.860 & 2.083 & 2.233 & 2.433 & 2.462 & 2.292 & 2.091 \\
\hline 1960 & 2.046 & 2.168 & 2.107 & 2.317 & 2.240 & 2.101 & 2.269 & 2.096 & 2.447 & 2.049 \\
\hline 1970 & 2.155 & $* 1.913$ & 2.666 & 2.479 & 2.815 & 2.527 & 2.089 & 2.431 & 2.309 & 2.277 \\
\hline 1980 & 2.540 & 2.429 & 2.077 & 2.381 & 2.374 & 2.289 & 2.042 & $* 1.857$ & $* 1.677$ & 2.835 \\
\hline 1990 & 2.180 & 2.022 & 2.310 & 2.644 & 2.800 & $* 1.940$ & 2.555 & 2.182 & $* 1.819$ & 2.238 \\
\hline 2000 & 2.519 & 2.740 & 2.156 & 2.371 & & & & & & \\
\hline
\end{tabular}

Os anos de *1950,*1971,*1987,*1988, *1995 e*1998, segundo estudo de BEZERRA (2004) foram anos de fenômenos oceânico-atmosféricos, de La Niña e El Niño, coprecipitações anuais coincidiram com o fenômeno El Niño e La Niña conforme BEZERRA (2004).

\section{Precipitação Ascendente}

A Tabela 2 representa a distribuição das chuvas anual do município de Porto Velho ordenada de forma ascendente dos 59 anos, na qual podemos quantificar em percentuais do total de ocorrência de chuva em um intervalo anual escolhida dentro da série analisada. Exemplificamos o intervalo de ocorrência de chuva entre 2.022 $\mathrm{mm}$ a $2.155 \mathrm{~mm}$, dos 59 anos representa $20 \%$ dos anos.
Conforme consta na Tabela 2, a precipitação abaixo de $2.000 \mathrm{~mm}$ distribuídos dentro da série correspondem apenas a 0,117 e 0,833 das precipitações que ocorreram acima de $2.000 \mathrm{~mm}$ anuais, ficando entre $2.022 \mathrm{~mm} /$ ano a $2.815 \mathrm{~mm}$ /ano. A amplitude entre a menos e a maior precipitação foi de $1.234 \mathrm{~mm}$ e o percentual de ocorrência de caso de precipitação anual abaixo ou acima da média $(2277,3 \mathrm{~mm})$ do período de 1945 a 2003 , teve seus percentuais de $48,3 \%$ e $51,7 \%$, a variabilidade da precipitação em torno da média do período tem uma diferença de apenas 3,4\% mostrando que tomando só a média como ponto de referência não é um bom indicador para a averiguação da variabilidade temporal dentro de uma série histórica, temos que utilizar o desvio padrão em torno da média para criar uma faixa de variabilidade mais significativa em torno dos casos extremos dentro de uma série, que será mais significativo.

\section{Frequência da Precipitação}

A distribuição dos conjuntos de dados de precipitação anual arranjados em classes e a mesma por números de ocorrência para um determinado intervalo de classe, serve de parâmetro quantificativo para saber quantas vezes um mesmo evento acontece, mediante a sua frequência. O município de Porto Velho que tem uma precipitação média de $2277,3 \mathrm{~mm}$, nos 59 anos de registro pluviômetros registrou os seus eventos extremos anual de 1.626 $\mathrm{mm}$ e $2.860 \mathrm{~mm}$.

A Tabela 3 mostra a distribuição de frequência dos totais anuais de chuva em um determinado intervalo de classe e dos 59 anos analisada os intervalos de precipitação das menores frequências encontram-se 
Tabela 2: Total de chuva de Porto Velho - RO, ordenado em ascendência $(n=N+1)$.

\begin{tabular}{|l|l|l|l|l|l|l|l|l|l|l|l|}
\hline $\mathbf{m}$ & $\mathbf{m} / \mathbf{n}$ & $\mathbf{X}$ & $\mathbf{m}$ & $\mathbf{m} / \mathbf{n}$ & $\mathbf{X}$ & $\mathbf{m}$ & $\mathbf{m} / \mathbf{n}$ & $\mathbf{X}$ & $\mathbf{m}$ & $\mathbf{m} / \mathbf{n}$ & $\mathbf{X}$ \\
\hline 1 & 0,017 & 1.626 & 16 & 0,267 & 2.091 & 31 & 0,517 & 2.292 & 46 & 0,767 & 2.479 \\
\hline 2 & 0,033 & 1.677 & 17 & 0,283 & 2.096 & 32 & 0,533 & 2.303 & 47 & 0,783 & 2.519 \\
\hline 3 & 0,050 & 1.819 & 18 & 0,300 & 2.101 & 33 & 0,550 & 2.309 & 48 & 0,800 & 2.527 \\
\hline 4 & 0,067 & 1.857 & 19 & 0,317 & 2.107 & 34 & 0,567 & 2.310 & 49 & 0,817 & 2.540 \\
\hline 5 & 0,083 & 1.913 & 20 & 0,333 & $\mathbf{2 . 1 5 5}$ & 35 & 0,583 & 2.317 & 50 & 0,833 & 2.555 \\
\hline 6 & 0,100 & 1.931 & 21 & 0,350 & 2.156 & 36 & 0,600 & 2.339 & 51 & 0,850 & 2.615 \\
\hline 7 & 0,117 & 1.940 & 22 & 0,367 & 2.168 & 37 & 0,617 & 2.371 & 52 & 0,867 & 2.644 \\
\hline 8 & 0,133 & $\mathbf{2 . 0 2 2}$ & 23 & 0,383 & 2.180 & 38 & 0,633 & 2.374 & 53 & 0,883 & 2.666 \\
\hline 9 & 0,150 & 2.024 & 24 & 0,400 & 2.182 & 39 & 0,650 & 2.381 & 54 & 0,900 & 2.713 \\
\hline 10 & 0,167 & 2.042 & 25 & 0,417 & 2.233 & 40 & 0,667 & 2.429 & 55 & 0,917 & 2.740 \\
\hline 11 & 0,183 & 2.046 & 26 & 0,433 & 2.238 & 41 & 0,683 & 2.431 & 56 & 0,933 & 2.800 \\
\hline 12 & 0,200 & 2.049 & 27 & 0,450 & 2.240 & 42 & 0,700 & 2.431 & 57 & 0,950 & 2.815 \\
\hline 13 & 0,217 & 2.077 & 28 & 0,467 & 2.269 & 43 & 0,717 & 2.433 & 58 & 0,967 & 2.835 \\
\hline 14 & 0,233 & 2.083 & 29 & 0,483 & 2.277 & 44 & 0,733 & 2.447 & 59 & 0,983 & 2.860 \\
\hline 15 & 0,250 & 2.089 & 30 & 0,500 & 2.289 & 45 & 0,750 & 2.462 & & & \\
\hline & & & & & & & & \\
\hline
\end{tabular}

Tabela 3: Distribuição de frequência dos totais anuais de chuva de Porto Velho - RO 45/03

\begin{tabular}{|l|l|llllllll|}
\hline $\begin{array}{l}\text { L i m i t e } \\
\text { Inferior }\end{array}$ & $\begin{array}{l}\text { L i m i t e } \\
\text { Superior }\end{array}$ & $\begin{array}{l}\text { Ponto Médio } \\
\text { (X) }\end{array}$ & Frequência & fa & fr & fra & fx & Fx $^{2}$ \\
\hline $1^{\text {a }} 1626$ & 1780 & 1703 & 2 & 2 & 0,034 & 0,034 & $\mathbf{3 4 0 6}$ & 5800418 \\
$2^{\text {a }} 1780$ & 1934 & 1857 & 4 & 6 & 0,068 & 0,102 & $\mathbf{7 4 2 8}$ & 13793796 \\
$3^{\text {a }} 1934$ & 2088 & 2011 & 11 & 17 & 0,186 & 0,288 & $\mathbf{2 2 1 2 1}$ & 44485331 \\
$4^{\text {a }} 2088$ & 2242 & 2165 & 10 & 27 & 0,169 & 0,458 & $\mathbf{2 1 6 5 0}$ & 46872250 \\
$5^{\text {a }} 2242$ & 2396 & 2319 & 12 & 39 & 0,203 & 0,661 & $\mathbf{2 7 8 2 8}$ & 64533132 \\
$6^{\text {a }} 2396$ & 2550 & 2473 & 10 & 49 & 0,169 & 0,831 & $\mathbf{2 4 7 3 0}$ & 61157290 \\
$7^{\text {a }} 2550$ & 2704 & 2627 & 4 & 53 & 0,068 & 0,898 & $\mathbf{1 0 5 0 8}$ & 27604516 \\
$8^{\text {a }} 2704$ & 2860 & 2782 & 6 & 59 & 0,102 & 1,000 & $\mathbf{1 6 6 9 2}$ & 46437144 \\
\hline Total & $\Sigma$ & & 59 & & 1,000 & 1,000 & $\mathbf{1 3 4 3 6 3}$ & 310683877 \\
\hline
\end{tabular}

$\sigma=282,1, \mu=2277,3, \mathrm{CV}=12,39 \%$

ente as $1^{\mathrm{a}}, 2^{\mathrm{a}}, 7^{\mathrm{a}}$ e $8^{\mathrm{a}}$ classes, com precipitação entre $1626 \mathrm{~mm}$ a $1934 \mathrm{~mm}$ e $2550 \mathrm{~mm}$ a $2850 \mathrm{~mm}$, sendo que as maiorias frequências vão da $3^{\mathrm{a}}$ a $6^{\mathrm{a}}$ classes com precipitação anual que variam de $2088 \mathrm{~mm}$ a 2549 mm, corresponde $72,9 \%$ dos casos.

A precipitação anual no município de Porto Velho não apresenta nenhuma tendência para aumento ou diminuição visto que as maiores frequências ficaram na faixa de variabilidade adotada que foi a média mais o desvio padrão e a média menos o desvio padrão, que computaram os valores de $2569,7 \mathrm{~mm}$ a 2003,5 $\mathrm{mm}$, equivalendo em $71,6 \%$ dos anos analisados,
$11,7 \%$ encontra-se abaixo da faixa de variabilidade e $15 \%$ encontra-se acima da faixa de variabilidade, conforme mostra a Tabela 2. Dos desvios de precipitação pluviométrica acima e abaixo da faixa de variabilidade adotada (ver Figura 13), 11,9\% ocorreram em anos de El Niño e 11,9 La Nina, conforme Tabela 4 e apenas $3,4 \%$ em anos que consideramos normal sem ocorrências de eventos oceânico-atmosféricos, porém acima e abaixo da faixa de variabilidade adotada, os restantes dos anos das séries 72,8\% encontram-se dentro da faixa considerada normal.

Segundo VAREJÃO-SILVA (2001) o El Niño 
Tabela 4: Desvios positivos e negativos de precipitação mais significativo do período de 1945 a 2003 com a ocorrência de El Niño/La

\begin{tabular}{l|l|l|l|l|l}
\multicolumn{5}{c}{ Niña do Município de Porto Velho - RO $(\mathrm{F}=$ Forte, $\mathrm{M}=$ Moderado, Fr = Fraco, DP = Desvio positivo e DN = desvio negativo. $)$} \\
\hline Anos & Desvio positivo (mm) & Fenômenos & Anos & Desvio negativo $(\mathrm{mm})$ & Fenômenos \\
\hline 1947 & 426 & El Niño - M & 1950 & 356 & La Niña - M \\
1949 & 328 & La Niña - F & 1952 & 661 & Normal \\
1953 & 573 & El Niño - Fr & 1971 & 374 & La Niña - Fr \\
1972 & 380 & El Niño - F & 1987 & 429 & El Niño - M \\
1974 & 528 & La Niña - F & 1988 & 610 & La Niña - F \\
1989 & 548 & La Niña - F & 1995 & 346 & La Niña - Fr \\
1993 & 357 & El Niño - F & 1998 & 468 & El Niño - F \\
1994 & 514 & El Niño - M & - & & - \\
2001 & 453 & Normal & - & & -
\end{tabular}

e a La Niña são exemplo incontestável de que o comportamento da atmosfera não pode ser entendido a nível regional (estudos restritos a pequenas áreas). A atmosfera age como um todo e determinados impulsos em uma área podem repercutir nos estados do tempo em regiões distintas, muitas vezes situadas a vários milhares de quilômetros dos locais onde tais impulsos ocorreram.

A distribuição da precipitação pluviométrica no período de 1945 a 2003 no município de Porto velho, dentro do ponto médio do intervalo de classe conforme consta na Tabela 3 e a Figura 16, está concentrada entre os intervalos de classe $2.088 \mathrm{~mm} / \mathrm{ano}$ a $2.473 \mathrm{~mm} / \mathrm{ano}$, dentro de uma frequência de ocorrência entre 10 a 12 de número de vezes de repetições de eventos de precipitações pluviométricas, contabilizando um total de $72,7 \%$ da frequência relativa neste intervalo de classe, mostrando que não há uma tendência para diminuição ou aumento da precipitação pluviométrica do município de Porto Velho, pois a maiorias dos eventos de precipitação pluviométricas anual encontram dentro do intervalo de variabilidade estabelecido como mostra a Figura 13. E os eventos considerados extremos para mais e para menos estão entre 6,8 a 10, 2 entre a frequência relativa para mais e 3,4 a 6,8 na frequência relativa para menos.

\section{CONCLUSÕES E RECOMENDAÇÕES}

De acordo com os objetivos propostos e os resultados obtidos, chegou-se às seguintes conclusões:

- Dos 59 anos analisados, apenas 11,7\% apresentaram valores de precipitação abaixo de $2.000 \mathrm{~mm} /$ ano e $86,6 \%$ acima de $2.000 \mathrm{~mm} / \mathrm{ano}$. As maiores frequências de precipitações estão entre $2.088 \mathrm{~mm} /$ ano a $2.550 \mathrm{~mm} / \mathrm{ano}$, que correspondem $72,9 \%$ e as menores frequências de precipitações estão entre $1.626 \mathrm{~mm}$ a 1780 $\mathrm{mm}$, correspondendo a $3,4 \%$ e as maiores frequências de precipitação estão entre $2.704 \mathrm{~mm}$ a $2.860 \mathrm{~mm}$ correspondendo a $10,1 \%$.

- Os eventos que consideramos extremos com variabilidade fora da faixa estabelecida com desvio de precipitação para mais e para menos $11,9 \%$ tiveram influência dos fenômenos El Niño e 11,9\% La Niña e 3,4\% em anos considerados normais sem a presença de tais fenômenos. Demonstrando que $27,2 \%$ das anomalias das precipitações do município de Porto Velho, 23,8\% sofrem influência marcante de eventos El Niño e La Nina.

- Na década de 50 ocorreram os maiores extremos de precipitação anual, sendo que o evento menor ocorreu em 1952, com 661 mm abaixo da média, ano considerado normal em relação aos fenômenos El Niño e La Niña e em 1953, com 573 mm acima da média anual de El Niño fraco. Nessa década o município de Porto Velho não tinha um contingente populacional como nas décadas de 70, 80 e 90, e o processo de desenvolvimento econômico tendo como matriz a agropecuária era praticamente 
inexistente.

- As variabilidades da precipitação mensal entre os decênios em todos os meses não ultrapassam $85 \mathrm{~mm}$ nos meses chuvosos e $28 \mathrm{~mm}$ nos meses de estiagem, mostrando que não há uma discrepância em relação à média do período de análise.

- Finalmente, sugere-se a realização de trabalho mais detalhado sobre precipitação do município de Porto Velho com ênfase na intensidade e duração, época do ano e frequência de ocorrência, para servir como auxilio em obras de drenagem, pontos de enchente e movimento de massa.

\section{REFERÊNCIAS}

AGUIAR, F. E. O. As Alterações Climáticas em Manaus no Século XX. 1995. 182f. Dissertação (Mestrado em Geografia) - Instituto de Geociências, Departamento de Geografia - PPGG, Universidade Federal do Rio de Janeiro, Rio de Janeiro, 1995.

BACK, A. J. Aplicação de análise Estatística para Identificação de Tendências Climáticas. Pesquisa agropecuária, Brasília, v. 36, n. 5, p 717 - 726, maio 2001.

EMBRAPA, Empresa Brasileira de Pesquisa Agropecuária. Sistema Brasileiro de Classificação de Solos. Brasília: Embrapa Produção de Informação, 1999.

BEZERRA, R. B. Balanço Hídrico em Porto VelhoRO, no Periodo de 1954 a 1993, através do Método de Thornthwaite e Mather (1955).1996, 83p. Monografia (Bacharelado em Geografia) - Universidade Federal de Rondônia - UNIR, Porto Velho - RO, 1996.

BEZERRA, R. B. Variabilidade espacial do balanço hídrico climatológico da região Norte do Brasil e influência do desmatamento em Porto Velho - RO. 2004. 75p. Dissertação (Mestrado em Geografia) - Universidade Federal de Campina Grande - PB UFCG, Campina Grande - PB, 2004.
IBGE. Fundação Instituto Brasileiro de Geografia e Estatístico. Estatística do Século XX do Brasil. Rio de Janeiro. IBGE CD-ROM, 2003.

IBGE. Fundação Instituto Brasileiro de Geografia e Estatístico . Cidades Rondônia Disponível em: http:// www.ibge.gov.br/cidadesat/topwindow.htm?1 Acesso em: 17 out. 2010.

INMET - Instituto Nacional de Meteorologia - Monitoramento das Estações Convencionais Comunicação pessoal via e-mail nadir.sales@inmet.gov.br em 26 out. 2003.

KÖPPEN, W. Climatología. México, Fondo de Cultura Económica. 1948.

MARENGO, J. A. Mudanças Climáticas Globais e Regionais: Avaliação atual do Brasil e Projeções de Cenários Climáticos do Futuro. Revista Brasileira de Meteorologia, v. 16, n.1, p. $01-18,2001$.

MENDONÇA, F.; DANNI-OLIVEIRA, I. M. Climatologia: Noções Básica e Clima do Brasil. São Paulo: Oficina de Texto, 2007.

NIMER, E.; BRANDÃO, A. M. P. M. Balanço Hídrico da Região dos Cerrados. Rio de Janeiro, infor. IBGE, 1989.

NIMER, E. Clima in Geografia do Brasil-Região Norte, v. 3, Rio de Janeiro: IBGE. Departamento de Recursos Naturais e Estudos Ambientais, 1991. p 46-54.

NOBRE, P.; MELO, A. B. C.. Variabilidade Climática Intrasazonal sobre o Nordeste do Brasil em 19982000. 2001.

NÓBREGA, R. S. Modelagem de Impactos do Desmatamento nos recursos Hídricos da Bacia Hidrográfica do Rio Jamari (RO) Utilizando Dados de Superficie e do TRMM. 2008. 213p. Tese (Doutorado em Meteorologia) - Unidade Acadêmica de Ciências Atmosféricas, Universidade Federal de Campina Grande - PB - UFCG, Campina Grande, 2008. 
QUADRO, M. F. L.; MACHADO, L. H. R.; CALBETE, N. O.; BATISTA, N. N. M.; SAMPAIO, G. Climatologia de Precipitação e Temperatura no Brasil. Climanálise Especial, v. único, p. 90-100, 1996.

RAO, V. B.; HADA, K. Characteristics of rainfall over Brazil: Annual Variations and connections with the Southern Oscillations. Theor. Appil. Climatol.42, p. $81-91,1990$.

RONDÔNIA. Plano Agroflorestal de RondôniaPLANAFLORO. Porto Velho: TECNOSSOLOS, Relatório Técnico, 2001.

SEDAM. Boletim Climático do Estado de Rondônia. v. 4. Secretaria de Desenvolvimento Ambiental do Estado de Rondônia, Porto Velho (RO), 2006.

SILVA, E. M.; RIBEIRO, A. G. As Tendências das Variações Climáticas na Cidade de Uberlândia-Mg (1981-2000). Caminhos de Geografia, v. 9, n. 12, p. 174-190, jun. 2004.

VAREJÃO-SILVA, M. A. Meteorologia e Climatologia. Brasília: INMET, Gráfica e Editora PAX, 2001.

VILLELA, S. M.; MATTOS, A. Hidrologia Aplicada.

São Paulo. McGraw-Hill do Brasil, 1975. 